\title{
Liver injury changed the biological characters of serum small extracellular vesicles and reprogramed hepatic macrophages in mice
}

\section{Xiufang Lv}

Department of Pathology, Medical School of Nantong University, Nantong, 226001, China; Key Laboratory of Microenvironment and Translational Cancer Research, Nantong, 226001, China https://orcid.org/0000-0003-2345-848X

\section{Anqi Zhang}

Department of Pathology, Medical School of Nantong University, Nantong, 226001, China. Key Laboratory of Microenvironment and Translational Cancer Research, Nantong, 226001, China

\section{Weiqi Liu}

Department of Pathology, Medical School of Nantong University, Nantong, 226001, China. Key Laboratory of Microenvironment and Translational Cancer Research, Nantong, 226001, China

\section{Min Zhao}

Department of Pathology, Medical School of Nantong University, Nantong, 226001, China. Key Laboratory of Microenvironment and Translational Cancer Research, Nantong, 226001, China Jing Li

Department of Pathology, Medical School of Nantong University, Nantong, 226001, China. Department of Pathology, National Cancer Center/National Clinical Research Center for Cancer/Cancer Hospital, Chinese Academy of Medical Sciences and Peking Union Medica

\section{Li He}

Department of Pathology, Medical School of Nantong University, Nantong, 226001, China. Department of Pathology, Nanjing Jinling Hospital, Nanjing University School of Medicine, Nanjing, 210093, China

\section{Li Cheng}

Department of Pathology, Medical School of Nantong University, Nantong, 226001, China. Department of Pathology, Affiliated Jiangyin Hospital of Southeast University Medical College, Jiangyin, 214400, China

\section{Yufeng Sun}

Department of Pathology, Medical School of Nantong University, Nantong, 226001, China. Key Laboratory of Microenvironment and Translational Cancer Research, Nantong, 226001, China

\section{Gang Qin}

Center for Liver Diseases, the Third Affiliated Hospital of Nantong University, Nantong, 226006, China

\section{Peng Lu}


Department of Pathology, Medical School of Nantong University, Nantong, 226001, China. Key Laboratory of Microenvironment and Translational Cancer Research, Nantong, 226001, China

\section{Yuhua Ji}

Key Laboratory of Neuroregeneration of Jiangsu and Ministry of Education, Nantong University, Nantong, 226001, China. Institute of Immunology, College of Life Science and Technology, Jinan University, Guangdong, 510632, China

\section{Juling Ji ( $\square$ jijuling@ntu.edu.cn )}

Department of Pathology, Medical School of Nantong University, Nantong, 226001, China. Key Laboratory of Microenvironment and Translational Cancer Research, Nantong, 226001, China https://orcid.org/0000-0001-6500-8052

\section{Research}

Keywords: microRNA, small RNA sequencing, biomarker, monocyte-derived macrophage, resident macrophage

Posted Date: August 25th, 2021

DOI: https://doi.org/10.21203/rs.3.rs-142242/v2

License: (c) (1) This work is licensed under a Creative Commons Attribution 4.0 International License. Read Full License 


\section{Abstract}

\section{BACKGROUND}

Serum small extracellular vesicles (sEVs) and their small RNA (sRNA) cargoes could be promising biomarkers for the diagnosis of liver injury. However, the dynamic changes in serum sEVs and their sRNA components during liver injury have not been well characterized. Given that hepatic macrophages can quickly clear intravenously injected SEVs, the effect of liver injury-related serum sEVs on hepatic macrophages deserves to be explored.

\section{AIM}

To identify the characteristics of serum sEVs and the sRNAs they contain during liver injury and explore their effects on hepatic macrophages.

\section{METHODS}

To identify serum sEV biomarkers for liver injury, we established a carbon tetrachloride-induced mouse liver injury model in C57BL/ 6 mice to simulate acute liver injury (ALI), chronic liver injury (CLI) and recovery. Serum sEVs were obtained and characterized by transmission electron microscopy and nanoparticle tracking analysis. Serum sEV sRNAs were profiled by sRNA sequencing. Differentially expressed microRNAs (miRNAs) were compared to mouse liver-enriched miRNAs and previously reported circulating miRNAs related to human liver diseases. The biological significance was evaluated by Ingenuity Pathway Analysis of altered sEV miRNAs and conditioned cultures of ALI serum sEVs with primary hepatic macrophages.

\section{RESULTS}

We found that both ALI and CLI changed the concentration and morphology of serum sEVs. The proportion of serum sEV miRNAs increased upon liver injury, with the liver as the primary contributor. The altered serum SEV miRNAs based on mouse studies were consistent with human liver disease-related circulating miRNAs. We established serum sEV miRNA signatures for ALI and CLI and a panel of miRNAs (miR-122-5p, miR-192-5p, and miR-22-3p) as a common marker for liver injury. The differential serum sEV miRNAs in ALI contributed mainly to liver steatosis and inflammation, while those in CLI contributed primarily to hepatocellular carcinoma and hyperplasia. ALI serum sEVs decreased both CD86 and CD206 expression in monocyte-derived macrophages but increased CD206 expression in resident macrophages in vitro.

\section{CONCLUSION}

Serum sEVs acquired different concentrations, sizes, morphologies and sRNA contents upon liver injury and could change the phenotype of liver macrophages. Serum sEVs therefore have good diagnostic and therapeutic potential for liver injury. 


\section{Background}

Because of its strategic location and biological functions, the liver is particularly susceptible to various pathogenic factors, including drugs, alcohol, and viruses. The initial insult induces acute liver injury (ALI) or even liver failure. Repeated or persistent insults will cause chronic liver injury (CLI), resulting in liver fibrosis and finally fatal cirrhosis ${ }^{[1,2]}$. Therefore, it is important to identify individuals with liver injury. However, liver injury does not always cause noticeable signs and symptoms. Aside from the widely used blood liver function tests on serum alanine aminotransferase (ALT) and aspartate aminotransferase (AST), which are not always restricted to liver injury ${ }^{[3]}$, there is still a need to explore specific and sensitive biomarkers. With the rapid progress in medical research, it is now possible and necessary to search for new biomarkers from serum small extracellular vesicles (sEVs).

Recently, serum sEVs have attracted tremendous interest due to their essential roles in intercellular communication and to their diagnostic and therapeutic potential ${ }^{[4]}$. The term sEVs refers to extracellular vesicles released by cells that are of relatively small size $(<200 \mathrm{~nm})$ and were previously regarded as exosomes ${ }^{[5]}$. The cargoes carried by sEVs represent a snapshot of the parental cells at the time of release and change depending on the physiological and pathological states ${ }^{[6,7]}$. In the liver, sEVs are released from both hepatocytes and nonparenchymal cells into the extracellular space and circulation. Several studies have reported that circulating sEV RNAs or proteins are abnormally expressed in the contexts of drug-induced liver injury, steatohepatitis, viral hepatitis, and hepatocellular carcinoma $(\mathrm{HCC})^{[6,8]}$.

MicroRNAs (miRNAs) are 22-24 nt small noncoding RNAs involved in posttranscriptional regulation and various biological processes ${ }^{[9]}$. Tissue-specific distribution is a key feature of miRNAs, making miRNAs good candidates as biomarkers or therapeutic targets for particular types of tissue injury ${ }^{[10-12]}$. Serum miRNAs have been studied in a variety of liver diseases ${ }^{[13]}$. However, compared to serum miRNAs, serum sEV miRNAs are well protected from RNA enzymes. Thus, serum sEV can serve as a more reliable miRNA pool ${ }^{[14]}$. We hypothesized that serum sEVs and their miRNA cargoes might reflect liver damage upon injury and could be promising biomarkers.

In the present study, we tried to determine the effects of liver injury on serum sEVs and the small RNAs (sRNAs) they transport; we were also interested in determining if there is any difference between acute and chronic injury. A study in this regard will aid in identification of potential serum sEV miRNA biomarkers. The dynamic changes in the number and morphology of serum sEVs and the sRNA components of serum sEVs were examined. The profiles of deregulated serum sEV miRNAs were obtained and compared to those of mouse liver enriched miRNAs and previously reported circulating miRNAs related to human liver diseases. To further evaluate the biological significance of serum sEVs upon liver injury, conditioned cultures of ALI serum sEVs and primary hepatic macrophages were carried out. 


\section{Materials And Methods}

\section{Animal studies}

Male C57BL/6 mice (8 weeks old) were purchased from the Shanghai Medical Laboratory Animal Center (Shanghai, China) and housed in the animal facility of Nantong University with temperature $25 \pm 2^{\circ} \mathrm{C}$ and $12 \mathrm{~h}$ light/dark cycle controls. All experimental protocols were approved by the Animal Ethics Committee of Nantong University. The animal care and experiments were performed in accordance with the relevant guidelines and regulations. For acute liver injury (ALI), mice were treated with a single dose of $\mathrm{CCL}_{4}(0.5$ $\mathrm{mL} / \mathrm{kg}$ intraperitoneal injection) dissolved in olive oil (1:9). The mice were sacrificed at 2 or 7 days. For chronic liver injury (CLI), mice were treated with $\mathrm{CCL}_{4}(0.5 \mathrm{~mL} / \mathrm{kg})$ or vehicle twice a week for eight weeks [15]. The mice were sacrificed $48 \mathrm{~h}$ after the last injection at 8 weeks or at 12 weeks (Figure 1A). Mice treated with the same volume of olive oil served as the controls for the ALI and CLI models, and 10-12 mice were used in each group. Blood or livers were collected from each group for further analyses.

\section{Liver function test and histopathologic examination}

Blood was collected by left ventricular puncture from mice and was left undisturbed for $1 \mathrm{~h}$ at $37^{\circ} \mathrm{C}$ and 2 $\mathrm{h}$ at $4^{\circ} \mathrm{C}$. Afterward, the samples were centrifuged at $1000 \times g$ for $10 \mathrm{~min}$ at $4^{\circ} \mathrm{C}$; the clear upper fractions were aliquoted and stored at $-80^{\circ} \mathrm{C}$. Serum ALT and AST levels were measured on an ADVIA 1800 autoanalyzer (Siemens Healthcare Diagnostics, Deerfield, IL, USA). The livers were preserved in $4 \%$ paraformaldehyde, paraffin-embedded and sectioned. The liver tissue sections were stained with hematoxylin and eosin (Beyotime Biotechnology, Shanghai, China) for routine histology and $0.1 \%$ Sirius Red (Sigma-Aldrich, St. Louis, MO, USA) for collagen evaluation.

\section{Mouse serum sEV isolation and characterization}

Exosome-enriched serum sEV fractions were precipitated using ultracentrifugation and an ExoQuick precipitation kit (System Biosciences Inc., Mountain View, CA, USA) ${ }^{[16]}$. The sizes and particle concentrations of the isolated serum sEVs were measured by nanoparticle tracking analysis (NTA, NanoSight NS300, Malvern, UK). Serum sEVs were visualized using transmission electron microscopy (TEM, HT7700, Hitachi Ltd., Tokyo, Japan). The expression of exosomal protein markers was determined by Western blot analysis. The details are provided in the Supporting Information.

\section{Small RNA library construction and deep sequencing}

Serum sEV small RNA-seq was conducted by BMK Biotech Co., Ltd. (Beijing, China) with biological replicates for each group.

\section{Sequencing data analysis and bioinformatic analysis}

The raw data were processed as described previously ${ }^{[16]}$. The trimmed sequencing reads were deposited in the European Nucleotide Archive (https://www.ebi.ac.uk/arrayexpress/experiments/E-MTAB-9462). 
Bioinformatic analysis of the differentially expressed serum sEV miRNAs was performed using Ingenuity Pathways Analysis (Qiagen, Valencia, CA, USA). The significance of enrichment for genes with particular biologically relevant functions was determined with a one-sided Fisher's exact test.

\section{Systematic review of abnormally expressed circulating miRNAs in human liver diseases}

The detailed procedure is provided in the Supporting Information.

\section{Isolation and culture of mouse hepatic macrophages}

Primary mouse hepatic macrophages were isolated from male C57BL/ 6 mouse livers by Percoll (GE Healthcare, Princeton, NJ, USA) density gradient centrifugation. Incubation of liver macrophages with mouse serum sEVs and subsequent multiple-color flow cytometric analysis were carried out. The details are provided in the Supporting Information.

\section{Statistical analysis}

Statistical analyses were performed with GraphPad Prism 7.0 software (GraphPad Software, Inc., La Jolla, CA). Quantitative data are reported as the mean \pm SD. Comparisons between groups were made by Student's t-test or one-way ANOVA. All P values were two-sided, and statistical significance was accepted for a value less than 0.05 . Except for the data from small RNA-seq experiments, which included two biological replicates for each group, the data provided in the present study were from three or more independent experiments.

Additional methods and details are provided in the Supporting Information.

\section{Results}

\section{Establishment and validation of $\mathrm{CCL}_{4}$-induced $\mathrm{ALI}$ and $\mathrm{CLI}$ in mice}

The mouse $\mathrm{CCL}_{4}$-induced $\mathrm{ALI}$ and recovery model and the mouse $\mathrm{CCL}_{4}$-induced $\mathrm{CLI}$ and recovery model were established and validated (Figure 1).

In acutely injured mice (at 2 days; 2D group), serum ALT and AST levels were increased, and hepatocyte necrosis and inflammatory cell infiltration were observed around the lobular central vein. After five days of recovery (at 7 days; 7D group), the elevated ALT and AST levels had returned to the baseline of the ALI control (AC) group, and the histological changes were also reversed (Figure 1B-C). For CLI, repeated $\mathrm{CCL}_{4}$ treatment induced a slight elevation in serum ALT (at 8 weeks; $8 \mathrm{~W}$ group), but the level returned to the baseline of the CLI control (CC) group by 4 weeks after cessation of $\mathrm{CCL}_{4}$ treatment (at 12 weeks; $12 \mathrm{~W}$ group) (Figure 1D). Although the ALT level change in CLI at $8 \mathrm{~W}$ was not as prominent as those in ALI at $2 \mathrm{D}$, the change was comparable to the ALT level changes reported by other study groups using the same mice CLI model ${ }^{[17,18]}$. Damaged hepatocytes and centrilobular contracture were observed in the livers of the CLI mice (8W), with mild inflammatory cell infiltration. Sirius Red staining 
showed obvious collagen deposition and pseudolobule formation in 8W CLI mice. These morphological changes were alleviated in recovered mice (12W) (Figure 1E).

\section{Identification and characterization of serum sEVs from ALI and CLI mice}

The isolated particles were spherical or cup-shaped, as observed by transmission electron microscopy (TEM) (Figure 2A). Exosomal protein markers, including CD63, CD81, and CD9, were all highly expressed (Figure 2B), as determined by Western blot analysis.

NTA showed that the mean diameter of the particles ranged from 90.2 to $127.8 \mathrm{~nm}$. The number of particles was higher in the 2D group but lower in the 7D group than in the control group, and the particle diameters were smaller in the 2D group but larger in the 7D group than in the control group. We also noticed that the size distribution of sEVs widened and that multiple peaks were present in the 7D group (Figure $2 \mathrm{C}$ ). For $\mathrm{CLI}$ mice, the particle concentrations in the $8 \mathrm{~W}$ and $12 \mathrm{~W}$ groups tended to be lower than those in the control group, although there were no significant differences. The size distribution of sEVs was expanded with multiple peaks in both the $8 \mathrm{~W}$ and $12 \mathrm{~W}$ groups, and the particle diameters were larger in both the $8 \mathrm{~W}$ and $12 \mathrm{~W}$ groups (Figure $2 \mathrm{D}$ ). TEM examination revealed that the multiple peaks reflected the aggregation or fusion of mouse serum SEVs present in 7D, 8W, and $12 \mathrm{~W}$ samples (Figure 2C-D).

These findings suggested that both ALI and CLI changed the number and morphology of mouse serum sEVs, and even when the visible histological changes of the liver had recovered in the 7D and 12W groups, the changes in particle number and morphology of serum sEVs persisted.

\section{Comparison of the SRNA components in serum sEVs from ALI and CLI mice}

Dramatic increases in both total RNA and sRNA levels in serum sEVs were observed upon ALI (2D) (Figure $3 A-B)$. For $C L I$, there was no significant difference in either sEV RNA or sRNA content among the groups. RNA sequencing (RNA-seq) and annotation revealed that each pair of biological repeats had at least $94.97 \%$ common sequences in clean reads (Figure S1). In the control groups (AC and CC), tRNA was the dominant sRNA species in serum sEVs, followed by rRNA and miRNA (Figure 3C). The most remarkable change in serum sEV sRNAs was the increase in miRNA proportion in both ALI and CLI mice. Compared to the control condition, ALI increased the proportion of miRNAs by more than fourfold, but the proportion returned to baseline by 7 days; the proportion of miRNAs increased by almost threefold in CLI mice but was partially restored by 12 weeks. With the increase in miRNA, the proportion of tRNA decreased (Figure 3D).

\section{miRNA expression profiles of serum sEVs from ALI and CLI mice}

For the ALI and CLI groups, 467 and 488 detectable sEVs (transcripts per million reads, TPM $\geq 5.0$ ) miRNAs were obtained, respectively. The biological replications were highly correlated in each group (Figure 4A). The RNA-seq data were further validated by qRT-PCR. Differentially expressed miRNAs with different abundances were selected for validation (Figure 4B). 
In total, 91 miRNAs were upregulated and 85 miRNAs were downregulated (fold change $\geq 2.0, \mathrm{P}<0.05$ ) in the 2D group compared with the AC group (Figure 4C, Table S1). The levels of most of these miRNAs had recovered to baseline levels in the 7D group, in which only eight upregulated miRNAs and 11 downregulated miRNAs were detected (fold change $\geq 2.0, \mathrm{P}<0.05$ ) (Figure $4 \mathrm{C}$, Table S2). The cumulative distribution frequency (CDF) was calculated by adding each proportion of miRNAs from most to least abundant. The plot shows that the top five upregulated miRNAs (miR-148a-3p, miR-122-5p, miR-192-5p, miR-22-3p, and miR-21a-5p) in the 2D group accounted for up to $84.27 \%$ of all detectable miRNAs (Figure 4C). Only 13 miRNAs were upregulated and six miRNAs were downregulated (fold change $\geq 2.0, P<0.05$ ) in the $8 \mathrm{~W}$ group compared with the CC group (Figure 4D, Table S3); in addition, 8 miRNAs were upregulated and three miRNAs were downregulated (fold change $\geq 2.0, \mathrm{P}<0.05$ ) in the $12 \mathrm{~W}$ group compared with the CC group (Figure 4D, Table S4). The CDF analysis showed that the top three upregulated miRNAs (miR-122-5p, miR-192-5p, and miR-22-3p) in the $8 \mathrm{~W}$ group constituted up to $43.48 \%$ of all detectable miRNAs (Figure S2). These findings suggested that both ALI and CLI induced changes in serum sEV miRNA composition. The changes were caused by the differential expression of a small number of miRNAs with high abundance.

The biological significance of these differentially expressed serum sEV miRNAs in liver injury was explored by Ingenuity Pathway Analysis (IPA, www.qiagen.com/ingenuity). For the 176 differentially expressed serum sEV miRNAs upon ALI, hepatic steatosis was the most significant hepatotoxic effect, followed by liver inflammation. For the 19 differentially expressed serum sEV miRNAs upon CLI, hepatocellular carcinoma was the most significant, followed by liver hyperplasia (Figure 4E).

\section{The liver is the main contributor to the differentially expressed serum sEV miRNAs during ALI and CLI}

We were interested in determining the contribution of liver cells to the changes in serum sEV miRNAs upon ALI and CLI. First, the liver miRNA expression profile for wild-type male C57BL/ 6 mice was established using BRB-Array Tool v4.6.0 (https://brb.nci.nih.gov) based on the RNA-seq data from GSE78792 (https://www.ncbi.nlm.nih.gov/geo/query/acc.cgi?acc=GSE78792) ${ }^{[19]}$ (Table S5). Differentially expressed serum sEV miRNAs from ALI and CLI mice were compared to the liver miRNA expression profile. Among the top ten most abundant liver miRNAs, seven were also among the top ten increased miRNAs in serum sEVs upon ALI (Table 1) and constituted up to $84.70 \%$ of the increased serum sEV miRNAs; for CLI, the levels of three were raised in serum sEVs. These three miRNAs were the top three miRNAs that increased upon CLI (Table 1) and constituted up to $60.56 \%$ of the total increased serum sEV miRNAs. These findings suggested the liver as the primary contributor to the upregulated serum sEV miRNAs during ALI and CLI and confirmed that the serum SEV miRNA test could be a reliable and sensitive way to monitor either acute or chronic liver injury.

\section{ALI and CLI signatures based on serum SEV miRNA profiling}

To identify serum sEV miRNA signatures for liver injury, we compared the differentially expressed serum sEV miRNAs in various stages of ALI and CLI. Compared to the levels in the vehicle control samples, eight 
miRNAs were upregulated and two miRNAs were downregulated significantly during the acute injury stage, and these changes were sustained through the chronic phase (fold change $\geq 2.0, P<0.05$ ); thus, they can serve as common liver injury signatures (Figure 5A). In addition, the levels of 166 miRNAs changed significantly during acute injury, while those of nine miRNAs changed dramatically during chronic injury. These miRNAs with high abundance (TPM_Mean >1000) have the potential to be acute or chronic liver injury signatures. The complete lists of these potential ALI and CLI serum sEV miRNA signatures are provided in Table S6. According to their abundance, up to the top 20 miRNAs are listed in Figure 5. For the recovery stage, 18 miRNAs were changed significantly in the ALI group, and 11 miRNAs were changed significantly in the CLI group (Figure 5B). Some of these miRNAs overlapped with differentially expressed serum sEV miRNAs in corresponding acute or chronic injury stages.

\section{Comparison of the potential ALI and CLI serum SEV miRNA signatures to human liver disease (HLD)- related circulating miRNAs}

To explore the biological significance of the ALI and CLI serum SEV miRNA signatures in HLDs, we performed a systematic review of abnormally expressed circulating miRNAs reported in various HLDs. In total, 299 and 257 studies were identified from the PubMed (https://pubmed.ncbi.nlm.nih.gov) and WOS (http://apps.webofknowledge.com/) databases, respectively (Figure 6A). Data were retrieved from 14 studies, including drug-induced liver injury (DILI) ${ }^{[20]}$, chronic hepatitis $\mathrm{B}(\mathrm{CHB})^{[21-25]}$, chronic hepatitis $\mathrm{C}$ $(\mathrm{CHC})^{[21,25-27]}$, nonalcoholic fatty liver disease (NAFLD) ${ }^{[28]}$, nonalcoholic steatohepatitis (NASH) ${ }^{[23]}$, liver cirrhosis (LC) ${ }^{[24,29,30]}$ and HCC ${ }^{[21-24,28-33]}$ studies. Details on the 14 articles are summarized in Table S7.

In total, 269 nonredundant abnormally expressed circulating miRNAs related to HLDs were extracted, and those that appeared $\geq$ four times were defined as high-frequency miRNAs (Figure 6A). Of the 30 highfrequency miRNAs (Table S7), 23 miRNAs were detected in ALI and CLI serum sEVs (Figure 6B), 14 miRNAs overlapped with ALI and CLI serum sEV signatures, and 12 miRNAs showed the same expression trend. Of the 12 miRNAs, three miRNAs (miR-122-5p, miR-192-5p, and miR-22-3p) were identified as being increased in both $\mathrm{ALI}$ and $\mathrm{CLI}$ mice and thus have the potential to serve as common signatures for either acute or chronic liver injury. The other 9 miRNAs were identified as ALI signatures (miR-21a-5p, miR-92a3p, miR-194-5p, miR-17-5p and miR-19b-3p were increased; miR-451a, miR-27a-3p, miR-26a-5p, and miR223-3p were decreased) and may reflect acute or active liver injury (Figure 6C). In addition, it was noteworthy that the four high-frequency circulating miRNAs reported in HLDs with decreased levels all exhibited decreased levels in serum sEVs upon ALI.

\section{Serum sEVs from liver injury mice induced hepatic macrophage reprogramming}

Primary mouse hepatic macrophages were isolated, purified and incubated with SYTO-labeled serum sEVs. After $24 \mathrm{~h}$, green fluorescence was observed in most macrophages in both the control and ALI (2D) serum sEV incubation groups (Figure 7A). These observations indicated that serum sEVs could be taken up by hepatic macrophages. Serum sEVs from ALI mice (2D) accelerated the adhesion of hepatic macrophages (Figure 7B). As determined by qRT-PCR, normal serum sEVs increased M1-like gene (IL-1B 
and TNFA) expression and decreased the expression of some M2-like genes (IL-10RB, CD163, ARG1, and CD206); ALI serum sEVs showed similar effects. However, compared to normal serum sEVs, ALI serum sEVs tended to decrease M1-like IL-1B but increase M2-like CD163 gene expression (Figure 7C).

In order to further dissect the effects of ALI serum SEVs on monocyte-derived and resident hepatic macrophage subgroups, multiple-color flow cytometric analyses were performed to assess the expression of M1-like CD86 and M2-like CD206 in CD11 b F4/80 Low monocyte-derived and CD11 b ${ }^{+} \mathrm{F} 4 / 80^{\text {High }}$ resident macrophages ( ${ }^{[34]}$ and our unpublished data). ALI serum sEVs decreased both CD86 and CD206 expression in the $\mathrm{CD} 11 \mathrm{~b}^{+} \mathrm{F} 4 / 80^{\text {Low }}$ subgroup but increased $\mathrm{CD} 206$ expression in the $\mathrm{CD} 11 \mathrm{~b}^{+} \mathrm{F} 4 / 80^{\mathrm{High}}$ subgroup (Figure 7D-E). These findings indicated that ALI serum sEVs might induce depolarization of $\mathrm{CD} 11 \mathrm{~b}^{+} \mathrm{F} 4 / 80^{\text {Low }}$ monocyte-derived macrophages but $\mathrm{M} 2$ differentiation of $\mathrm{CD} 11 \mathrm{~b}^{+} \mathrm{F} 4 / 80^{\text {High }}$ resident macrophages.

\section{Discussion}

A growing number of studies have suggested the diagnostic value of serum sEV content for liver injury, especially miRNAs ${ }^{[6,8]}$. To explore potential serum sEV miRNA biomarkers for liver injury, we simulated the complex processes of liver injury and recovery in $\mathrm{CCL}_{4}$-induced acute and chronic liver injury mouse models. The 2D group and $8 \mathrm{~W}$ group represented acute and chronic liver injury, respectively, while the 7D group and $12 \mathrm{~W}$ group represented the recovery stage of acute and chronic liver injury. The ALT and AST levels elevated in the 2D group and $8 \mathrm{~W}$ group, and returned to the baseline of the control groups (Figure 1B, D). Moreover, the histological changes also reversed in the $7 \mathrm{D}$ and $12 \mathrm{~W}$ recovery groups (Figure 1C, E), which indicated that the acute and chronic liver injury and recovery models were well established.

Unexpectedly, aside from differentially expressed miRNAs, we found that the concentration, size, and morphology of serum sEVs might be essential features in liver injury (Figure 2). The number of serum sEVs increased upon ALI, which has been reported in human alcoholic hepatitis and alcoholic liver injury mouse models ${ }^{[35-37]}$. Furthermore, we found sustained decreases in serum sEV number during the chronic injury stage and the recovery stage for both acute and chronic liver injury. Moreover, serum sEVs became smaller upon ALI but enlarged during ALI recovery and the CLI stage, and the increased size persisted through the $\mathrm{CLI}$ recovery stage, at which time there were multiple peaks, as observed by NTA. Interestingly, aggregation of serum sEV particles was observed in samples from the ALI recovery stage, CLI stage, and CLI recovery stage by TEM, which explained the multiple peaks and increased particle size found by NTA. We propose that the aggregation of sEVs might reflect membrane damage of extracellular vesicles following liver injury. Thus, changes in serum sEV concentration, size, and morphology are well connected to a particular stage of liver injury and could provide diagnostic clues. In addition, the changes in serum sEVs persisted even when liver function and visible histopathological changes were restored; thus, they could be useful to trace recent liver injury. 
Then, we showed that ALI and CLI altered the sRNA levels and components in serum sEVs. For ALI, both the total RNA and sRNA levels of serum sEVs increased significantly, and the proportion of miRNAs in sRNA also increased. Although there were no significant increases in RNA or sRNA levels for CLI, the proportion of miRNAs increased significantly. Compared to CLI, ALI significantly changed more miRNA species (176 vs. 19 , fold change $\geq 2.0, \mathrm{P}<0.05$ ). The increased proportion of miRNAs upon liver injury was mainly attributable to a few highly abundant miRNAs. To traceback the primary source of the increased serum sEV miRNAs, the highly abundant miRNAs detected in serum sEVs from ALI and CLI mice were evaluated in the livers of normal male C57BL/ 6 mice. The miRNAs with the highest abundance were all liver-enriched miRNAs (Table 1). We propose that the liver is the main contributor to the elevations in miRNAs in serum sEVs for both acute and chronic liver injury. Thus, serum sEVs carry the miRNA messages released from the injured liver, and examining the serum sEV miRNAs could be a reliable way to monitor either acute or chronic liver injury.

By comparing the expression profiles of serum sEV miRNAs in various stages of ALI and CLI, we obtained a list of miRNAs that can be used as common liver injury signatures as well as the miRNA signatures for $\mathrm{ALI}, \mathrm{CLI}$, and the recovery stages. However, these signatures were obtained from mouse models and need to be validated in human patients. Hence, we carried out a systematic review of previously published studies and obtained 30 miRNAs that were highly correlated with human liver disease, including DILI, CHB, CHC, NAFLD, NASH, and liver cirrhosis (Figure 6). Of these 30 miRNAs, three miRNAs (miR-122-5p, miR-192-5p, and miR-22-3p) were identified as common injury signatures that were increased in both ALI and CLI mice. Although these three miRNAs have been studied separately $[24,36,38$, ${ }^{39]}$, here, for the first time, they were combined as a universal signature for either acute or chronic liver injury. Nine miRNAs were identified as ALI signatures. Along with the five increased miRNAs (miR-21a-5p, miR-92a-3p, miR-194-5p, miR-17-5p, and miR-19b-3p), four miRNAs were decreased (miR-451a, miR-27a$3 p$, miR-26a-5p, and miR-223-3p), the same four miRNAs that are frequently reported to be decreased in human liver disease (Figure 6). Although the human data were mostly from patients with chronic liver injury, the overlapping ALI signatures might reflect active lesions. Based on serum sEV miRNAs, we established common signatures for liver injury and specific signatures for acute/active liver injury.

The biological significance of the alterations in SEV miRNAs upon liver injury was explored by IPA. The top hepatotoxicity-related functions of the altered serum sEV miRNAs in ALI were hepatic steatosis and liver inflammation, while the altered miRNAs carried by serum sEVs from CLI were linked primarily to hepatocellular carcinoma, followed by liver hyperplasia. These findings are consistent with the clinical findings that ALI mainly causes inflammation and that sustained damage increases the risk of carcinoma ${ }^{[40,41]}$. Hepatic steatosis reflects fatty degeneration that is typically caused by $\mathrm{CCL}_{4}{ }^{[42]}$. These findings reveal the pathological importance of serum sEVs during the processes of acute and chronic liver injury.

It has been reported that hepatic macrophages can take up serum sEVs and play essential roles in the clearance of intravenously injected sEVs from the systemic circulation ${ }^{[43,44]}$. We were interested in 
determining the effects of liver injury-related serum sEVs on hepatic macrophages and whether these effects could aggravate liver damage or play a protective role. We found that in vitro, ALI serum sEVs could be taken up by hepatic macrophages and promote macrophage adhesion. Furthermore, ALI serum sEVs tended to decrease M1-like gene expression, such as IL-1B and TNFA expression, and increase M2like gene expression, including CD163 expression. However, the trends were not consistent. Considering that hepatic macrophages are heterogeneous populations composed of two subgroups, including resident macrophages and circulating monocyte-derived macrophages ${ }^{[34]}$, we propose that macrophages of different origins might react inconsistently to ALI serum sEVs. The expression of M1 (CD86) and M2 (CD206) polarization signatures on the two subgroups of hepatic macrophages was examined by flow cytometry. ALI serum sEVs induced depolarization of $C D 11 b^{+} F 4 / 80^{L o w}$ monocyte-derived macrophages but induced $\mathrm{M} 2$ differentiation of $\mathrm{CD} 11 \mathrm{~b}^{+} \mathrm{F} 4 / 80^{\mathrm{High}}$ resident macrophages. We propose that the changes in serum sEVs upon ALI might alleviate liver damage by depolarizing monocyte-derived macrophages and educating resident hepatic macrophages to transform into M2-like cells.

\section{Conclusion}

In conclusion, we found that the concentration, size, and morphology of serum sEV particles were essential features for liver injury. We established specific serum sEV miRNA signatures for different liver injury stages and created a list of miRNAs that can be used as common liver injury biomarkers. The altered ALI and CLI serum sEV miRNAs were connected to diverse liver pathological processes. ALI serum $s E V s$ reprogrammed hepatic macrophage subgroups differently. Serum sEVs not only have good diagnostic potential but also could be used to ameliorate liver injury. However, the diagnostic and therapeutic potential of these altered serum sEVs upon liver injury deserves further study.

\section{Article Highlights}

\section{Research background}

Both acute liver injury (ALI) and chronic liver injury (CLI) do not always cause noticeable signs and symptoms. Serum small extracellular vesicles (SEVs) have attracted tremendous interest due to their essential roles in intercellular communication and their diagnostic and therapeutic potential. The cargoes carried by sEVs represent a snapshot of the parental cells and change depending on the physiological and pathological states.

\section{Research motivation}

Serum sEVs and their small RNA (sRNA) cargoes could be promising biomarkers for the diagnosis of liver injury.

\section{Research objectives}


The present study aimed to characterize the dynamic changes of serum sEVs and their sRNA components during liver injury and to explore the effect of liver injury-related serum sEVs on hepatic macrophages.

\section{Research methods}

Male C57BL/6 mice were treated with carbon tetrachloride $\left(\mathrm{CCL}_{4}\right)$ to establish a mouse liver injury model for simulating ALI, CLI, and recovery. Serum sEVs were obtained and characterized by transmission electron microscopy and nanoparticle tracking analysis. Serum sEV sRNAs were profiled by sRNA sequencing. Differentially expressed microRNAs (miRNAs) were compared to mouse liver-enriched miRNAs and previously reported circulating miRNAs related to human liver diseases. The biological significance was evaluated by Ingenuity Pathway Analysis of the altered sEV miRNAs and conditioned cultures of ALI serum sEVs with primary hepatic macrophages.

\section{Research results}

Both $\mathrm{ALI}$ and CLI changed the concentration and morphology of serum sEVs. The proportion of serum sEV miRNAs increased upon liver injury, with the liver as the primary contributor. The altered serum sEV miRNAs based on mouse study were consistent with human liver disease-related circulating miRNAs. We established serum sEV miRNA signatures for ALI and CLI and a panel of miRNAs (miR-122-5p, miR-192$5 p$, and miR-22-3p) as a common marker for liver injury. ALI serum sEVs decreased both CD86 and CD206 expression in monocyte-derived macrophages but increased CD206 expression in resident macrophages in vitro.

\section{Research conclusions}

Serum sEVs acquired different concentrations, sizes, morphologies, and sRNA contents upon diverse liver injured pathological processes. ALI serum sEVs reprogrammed hepatic macrophage subgroups differently.

\section{Research perspectives}

Serum sEVs have good diagnostic and therapeutic potential for liver injury.

\section{Acknowledgements}

The authors would like to acknowledge Yi Shen for the statistical review.

\section{References}

1 Reuben A, Koch DG, Lee WM, Acute Liver Failure Study G. Drug-induced acute liver failure: results of a U.S. multicenter, prospective study. Hepatology 2010; 52(6): 2065-2076 [PMID: 20949552 PMCID: 3992250 DOI: 10.1002/hep.23937] 
2 Kullak-Ublick GA, Andrade RJ, Merz M, End P, Benesic A, Gerbes AL, Aithal GP. Drug-induced liver injury: recent advances in diagnosis and risk assessment. Gut 2017; 66(6): 1154-1164 [PMID: WOS:000401136700021 DOI: 10.1136/gutjnl-2016-313369]

3 Nathwani RA, Pais S, Reynolds TB, Kaplowitz N. Serum alanine aminotransferase in skeletal muscle diseases. Hepatology 2005; 41(2): 380-382 [PMID: 15660433 DOI: 10.1002/hep.20548]

$4 \quad$ Hubbell CL, Mankes RF, Reid LD. A small dose of morphine leads rats to drink more alcohol and achieve higher blood alcohol concentrations. Alcohol Clin Exp Res 1993; 17(5): 1040-1043 [PMID: 8279664 DOI: 10.1111/j.1530-0277.1993.tb05661.x]

5 Théry C, Witwer KW, Aikawa E, Alcaraz MJ, Anderson JD, Andriantsitohaina R, Antoniou A, Arab T, Archer F, et al. Minimal information for studies of extracellular vesicles 2018 (MISEV2018): a position statement of the International Society for Extracellular Vesicles and update of the MISEV2014 guidelines. Journal of extracellular vesicles 2018; 7(1): 1535750 [PMID: 30637094 DOI: $10.1080 / 20013078.2018 .1535750]$

6 Szabo G, Momen-Heravi F. Extracellular vesicles in liver disease and potential as biomarkers and therapeutic targets. Nature reviews Gastroenterology \& hepatology 2017; 14(8): 455-466 [PMID: 28634412 PMCID: 6380505 DOI: 10.1038/nrgastro.2017.71]

7 Valadi H, Ekstrom K, Bossios A, Sjostrand M, Lee JJ, Lotvall JO. Exosome-mediated transfer of mRNAs and microRNAs is a novel mechanism of genetic exchange between cells. Nat Cell Biol 2007; 9(6): 654-U672 [PMID: WOS:000246922700010 DOI: 10.1038/ncb1596]

8 Urban SK, Mocan T, Sanger H, Lukacs-Kornek V, Kornek M. Extracellular Vesicles in Liver Diseases: Diagnostic, Prognostic, and Therapeutic Application. Seminars in liver disease 2019; 39(1): 70-77 [PMID: 30654391 DOI: 10.1055/s-0038-1676122]

9 Bartel DP. MicroRNAs: genomics, biogenesis, mechanism, and function. Cell 2004; 116(2): 281 297 [PMID: 14744438 DOI: 10.1016/s0092-8674(04)00045-5]

10 Erdos Z, Barnum JE, Wang E, DeMaula C, Dey PM, Forest T, Bailey WJ, Glaab WE. Evaluation of the Relative Performance of Pancreas-Specific MicroRNAs in Rat Plasma as Biomarkers of Pancreas Injury. Toxicological sciences : an official journal of the Society of Toxicology 2020; 173(1): 5-18 [PMID: 31504967 DOI: $10.1093 /$ toxsci/kfz184]

11 Laterza OF, Lim L, Garrett-Engele PW, Vlasakova K, Muniappa N, Tanaka WK, Johnson JM, Sina JF, Fare TL, et al. Plasma MicroRNAs as sensitive and specific biomarkers of tissue injury. Clinical chemistry 2009; 55(11): 1977-1983 [PMID: 19745058 DOI: 10.1373/clinchem.2009.131797]

12 Sun P, Liu DZ, Jickling GC, Sharp FR, Yin KJ. MicroRNA-based therapeutics in central nervous system injuries. Journal of cerebral blood flow and metabolism : official journal of the International 
Society of Cerebral Blood Flow and Metabolism 2018; 38(7): 1125-1148 [PMID: 29708005 PMCID: Pmc6434449 DOI: 10.1177/0271678x18773871]

13 Wang XW, Heegaard NHH, Orum H. MicroRNAs in Liver Disease. Gastroenterology 2012; 142(7): 1431-1443 [PMID: WOS:000304778700020 DOI: 10.1053/j.gastro.2012.04.007]

14 Cheng L, Sharples RA, Scicluna BJ, Hill AF. Exosomes provide a protective and enriched source of miRNA for biomarker profiling compared to intracellular and cell-free blood. Journal of extracellular vesicles 2014; 3 [PMID: 24683445 PMCID: Pmc3968297 DOI: 10.3402/jev.v3.23743]

15 Gu L, Deng WS, Sun XF, Zhou H, Xu Q. Rapamycin ameliorates CCl4-induced liver fibrosis in mice through reciprocal regulation of the Th17/Treg cell balance. Molecular medicine reports 2016; 14(2): 1153-1161 [DOI: 10.3892/mmr.2016.5392]

16 Zhao F, Cheng L, Shao Q, Chen Z, Lv X, Li J, He L, Sun Y, Ji Q, et al. Characterization of serum small extracellular vesicles and their small RNA contents across humans, rats, and mice. Scientific reports 2020; 10(1): 4197 [DOI: 10.1038/s41598-020-61098-9]

17 Kwon HJ, Won YS, Park O, Chang B, Duryee MJ, Thiele GE, Matsumoto A, Singh S, Abdelmegeed MA, et al. Aldehyde dehydrogenase 2 deficiency ameliorates alcoholic fatty liver but worsens liver inflammation and fibrosis in mice. Hepatology 2014; 60(1): 146-157 [PMID: 24492981 PMCID: 4077916 DOI: 10.1002/hep.27036]

18 Ma X, Luo Q, Zhu H, Liu X, Dong Z, Zhang K, Zou Y, Wu J, Ge J, et al. Aldehyde dehydrogenase 2 activation ameliorates $\mathrm{CCl} 4$-induced chronic liver fibrosis in mice by up-regulating Nrf2/HO-1 antioxidant pathway. Journal of cellular and molecular medicine 2018; 22: 3965-3978 [PMID: 29799157 PMCID: 6050510 DOI: $10.1111 / \mathrm{jcmm} .13677]$

19 Langfelder P, Gao F, Wang N, Howland D, Kwak S, Vogt TF, Aaronson JS, Rosinski J, Coppola G, et al. MicroRNA signatures of endogenous Huntingtin CAG repeat expansion in mice. PLoS One 2018; 13(1): e0190550 [PMID: 29324753 PMCID: 5764268 DOI: 10.1371/journal.pone.0190550]

20 Russo MW, Steuerwald N, Norton HJ, Anderson WE, Foureau D, Chalasani N, Fontana RJ, Watkins $\mathrm{PB}$, Serrano J, et al. Profiles of miRNAs in serum in severe acute drug induced liver injury and their prognostic significance. Liver international : official journal of the International Association for the Study of the Liver 2017; 37(5): 757-764 [PMID: 27860186 PMCID: Pmc5502673 DOI: 10.1111/liv.13312]

21 Li LM, Hu ZB, Zhou ZX, Chen X, Liu FY, Zhang JF, Shen HB, Zhang CY, Zen K. Serum microRNA profiles serve as novel biomarkers for HBV infection and diagnosis of HBV-positive hepatocarcinoma. Cancer research 2010; 70(23): 9798-9807 [PMID: 21098710 DOI: 10.1158/00085472.can-10-1001] 
22 Wang Y, Gao Y, Shi W, Zhai D, Rao Q, Jia X, Liu J, Jiao X, Du Z. Profiles of differential expression of circulating microRNAs in hepatitis B virus-positive small hepatocellular carcinoma. Cancer biomarkers : section A of Disease markers 2015; 15(2): 171-180 [PMID: 25519019 DOI: 10.3233/cbm-140451]

23 Sohn W, Kim J, Kang SH, Yang SR, Cho JY, Cho HC, Shim SG, Paik YH. Serum exosomal microRNAs as novel biomarkers for hepatocellular carcinoma. Experimental \& molecular medicine 2015; 47: e184 [PMID: 26380927 PMCID: Pmc4650928 DOI: 10.1038/emm.2015.68]

24 Zhou J, Yu L, Gao X, Hu J, Wang J, Dai Z, Wang J-F, Zhang Z, Lu S, et al. Plasma MicroRNA Panel to Diagnose Hepatitis B Virus-Related Hepatocellular Carcinoma. Journal of Clinical Oncology 2011; 29(36): 4781-4788 [PMID: WOS:000298142100013 DOI: 10.1200/jco.2011.38.2697]

25 Murakami Y, Toyoda H, Tanahashi T, Tanaka J, Kumada T, Yoshioka Y, Kosaka N, Ochiya T, Taguchi Yh. Comprehensive miRNA Expression Analysis in Peripheral Blood Can Diagnose Liver Disease. Plos One 2012; 7(10) [PMID: WOS:000310600500114 DOI: 10.1371/journal.pone.0048366]

26 Murakami Y, Tanahashi T. Analysis of circulating microRNA by microarray in liver disease. Methods in molecular biology (Clifton, NJ) 2013; 1024: 173-182 [PMID: 23719950 DOI: 10.1007/978-1-62703-453-1_13]

27 Santangelo L, Bordoni V, Montaldo C, Cimini E, Zingoni A, Battistelli C, D'Offizi G, Capobianchi MR, Santoni A, et al. Hepatitis $C$ virus direct-acting antivirals therapy impacts on extracellular vesicles microRNAs content and on their immunomodulating properties. Liver international : official journal of the International Association for the Study of the Liver 2018; 38(10): 1741-1750 [PMID: 29359389 DOI: 10.1111/liv.13700]

28 Tan Y, Ge G, Pan T, Wen D, Gan J. A pilot study of serum microRNAs panel as potential biomarkers for diagnosis of nonalcoholic fatty liver disease. PLoS One 2014; 9(8): e105192 [PMID: 25141008 PMCID: Pmc4139327 DOI: 10.1371/journal.pone.0105192]

29 Gui J, Tian Y, Wen X, Zhang W, Zhang P, Gao J, Run W, Tian L, Jia X, et al. Serum microRNA characterization identifies miR-885-5p as a potential marker for detecting liver pathologies. Clinical science (London, England : 1979) 2011; 120(5): 183-193 [PMID: 20815808 PMCID: Pmc2990200 DOI: 10.1042/cs20100297]

30 Fornari F, Ferracin M, Trere D, Milazzo M, Marinelli S, Galassi M, Venerandi L, Pollutri D, Patrizi C, et al. Circulating microRNAs, miR-939, miR-595, miR-519d and miR-494, Identify Cirrhotic Patients with HCC. PLoS One 2015; 10(10): e0141448 [PMID: 26509672 PMCID: Pmc4624953 DOI: 10.1371/journal.pone.0141448]

31 Chiu LY, Kishnani PS, Chuang TP, Tang CY, Liu CY, Bali D, Koeberl D, Austin S, Boyette K, et al. Identification of differentially expressed microRNAs in human hepatocellular adenoma associated with 
type I glycogen storage disease: a potential utility as biomarkers. Journal of gastroenterology 2014; 49(8): 1274-1284 [PMID: 24129885 DOI: 10.1007/s00535-013-0890-2]

32 Lu L, Guo D, Chen X, Xiong W, Jie S, Li H. Abnormal miRNAs Targeting Chromosome Open Reading Frame Genes were Enriched in Microvesicles Derived from the Circulation of HCC. Biochemical genetics 2016; 54(2): 120-133 [PMID: 26615601 DOI: 10.1007/s10528-015-9705-x]

33 Xue X, Zhao Y, Wang X, Qin L, Hu R. Development and validation of serum exosomal microRNAs as diagnostic and prognostic biomarkers for hepatocellular carcinoma. J Cell Biochem 2019; 120(1): 135142 [PMID: 30238497 DOI: 10.1002/jcb.27165]

34 Tacke F, Zimmermann HW. Macrophage heterogeneity in liver injury and fibrosis. Journal of hepatology 2014; 60(5): 1090-1096 [PMID: 24412603 DOI: 10.1016/j.jhep.2013.12.025]

35 Momen-Heravi F, Saha B, Kodys K, Catalano D, Satishchandran A, Szabo G. Increased number of circulating exosomes and their microRNA cargos are potential novel biomarkers in alcoholic hepatitis. $J$ Trans/ Med 2015; 13 [PMID: WOS:000359338000001DOI: 10.1186/S12967-015-0623-9]

36 Momen-Heravi F, Bala S, Kodys K, Szabo G. Exosomes derived from alcohol-treated hepatocytes horizontally transfer liver specific miRNA-122 and sensitize monocytes to LPS. Sci Rep 2015; 5: 9991 [PMID: 25973575 DOI: 10.1038/srep09991]

37 Eguchi A, Lazaro RG, Wang J, Kim J, Povero D, Willliams B, Ho SB, Stärkel P, Schnabl B, et al. Extracellular vesicles released by hepatocytes from gastric infusion model of alcoholic liver disease contain a MicroRNA barcode that can be detected in blood. Hepatology 2017; 65(2): 475-490 [PMID: 27639178 PMCID: Pmc5407075 DOI: 10.1002/hep.28838]

38 Motawi TK, Mohamed MR, Shahin NN, Ali MAM, Azzam MA. Time-course expression profile and diagnostic potential of a miRNA panel in exosomes and total serum in acute liver injury. The International Journal of Biochemistry \& Cell Biology 2018; 100: 11-21 [DOI: https://doi.org/10.1016/j.biocel.2018.05.002]

39 Arataki K, Hayes CN, Akamatsu S, Akiyama R, Abe H, Tsuge M, Miki D, Ochi H, Hiraga N, et al. Circulating microRNA-22 correlates with microRNA-122 and represents viral replication and liver injury in patients with chronic hepatitis B. Journal of Medical Virology 2013; 85(5): 789-798 [PMID: WOS:000316971500006 DOI: 10.1002/jmv.23540]

40 Michelotti GA, Machado MV, Diehl AM. NAFLD, NASH and liver cancer. Nature reviews Gastroenterology \& hepatology 2013; 10(11): 656-665 [PMID: 24080776 DOI: 10.1038/nrgastro.2013.183]

41 Nguyen VT, Law MG, Dore GJ. Hepatitis B-related hepatocellular carcinoma: epidemiological characteristics and disease burden. Journal of viral hepatitis 2009; 16(7): 453-463 [PMID: 19302335 DOI: 
42 Recknagel RO, Glende EA, Jr., Dolak JA, Waller RL. Mechanisms of carbon tetrachloride toxicity. Pharmacology \& therapeutics 1989; 43(1): 139-154 [PMID: MEDLINE:2675128 DOI: 10.1016/0163-7258(89)90050-8]

43 Imai T, Takahashi Y, Nishikawa M, Kato K, Morishita M, Yamashita T, Matsumoto A, Charoenviriyakul C, Takakura Y. Macrophage-dependent clearance of systemically administered B16BL6derived exosomes from the blood circulation in mice. Journal of extracellular vesicles 2015; 4: 26238 [PMID: 25669322 PMCID: Pmc4323410 DOI: 10.3402/jev.v4.26238]

44 Matsumoto A, Takahashi Y, Chang HY, Wu YW, Yamamoto A, Ishihama Y, Takakura Y. Blood concentrations of small extracellular vesicles are determined by a balance between abundant secretion and rapid clearance. Journal of extracellular vesicles 2020; 9(1): 1696517 [PMID: 31807238 PMCID: 6882433 DOI: 10.1080/20013078.2019.1696517]

\section{Footnotes}

Institutional review board statement: The study was reviewed and approved by the Nantong University Medical School Ethics Committee (License No. NTUMEC2017-20).

Institutional animal care and use committee statement: All experimental protocols were approved by the Animal Ethics Committee of Nantong University (License No. S20170102-011). The animal care and experiments were performed in accordance with the relevant guidelines and regulations.

Conflict-of-interest statement: The authors declare no competing interests.

Data sharing statement: All data used for the present study are presented in the main manuscript and its supplementary information files.

ARRIVE guidelines statement: The authors have read the ARRIVE guidelines, and the manuscript was prepared and revised according to the ARRIVE guidelines.

\section{Tables}

Table 1 Liver-enriched miRNAs ranked highly among serum sEV miRNAs from ALI and CLI model mice 


\begin{tabular}{lclcllc}
\hline GSE78792 Liver & Rank & \multicolumn{1}{c}{ ALI } & ALI Rank & CLI & CLI Rank \\
\hline miR-192 & 1 & miR-192-5p & 3 & miR-192-5p & 2 \\
\hline miR-22 & 2 & miR-22-3p & 4 & miR-22-3p & 3 \\
\hline miR-30a & 3 & miR-30a-5p & 7 & $\mathrm{n}$ & $\mathrm{n}$ \\
\hline miR-148a & 4 & miR-148a-3p & 1 & $\mathrm{n}$ & $\mathrm{n}$ \\
\hline miR-21a & 5 & miR-21a-5p & 5 & $\mathrm{n}$ & $\mathrm{n}$ \\
\hline miR-26a-2 & 6 & miR-26a-5p & 105 & $\mathrm{n}$ & $\mathrm{n}$ \\
\hline miR-122 & 7 & miR-122-5p & 2 & miR-122-5p & 1 \\
\hline miR-10a & 8 & $\mathrm{n}$ & $\mathrm{n}$ & $\mathrm{n}$ & $\mathrm{n}$ \\
\hline miR-143 & 9 & miR-143-3p & 92 & $\mathrm{n}$ & $\mathrm{n}$ \\
\hline miR-27b & 10 & miR-27b-3p & 10 & $\mathrm{n}$ & $\mathrm{n}$ \\
\hline
\end{tabular}

ALI: acute liver injury; CLI: chronic liver injury; n: not applicable.

Figures 
A

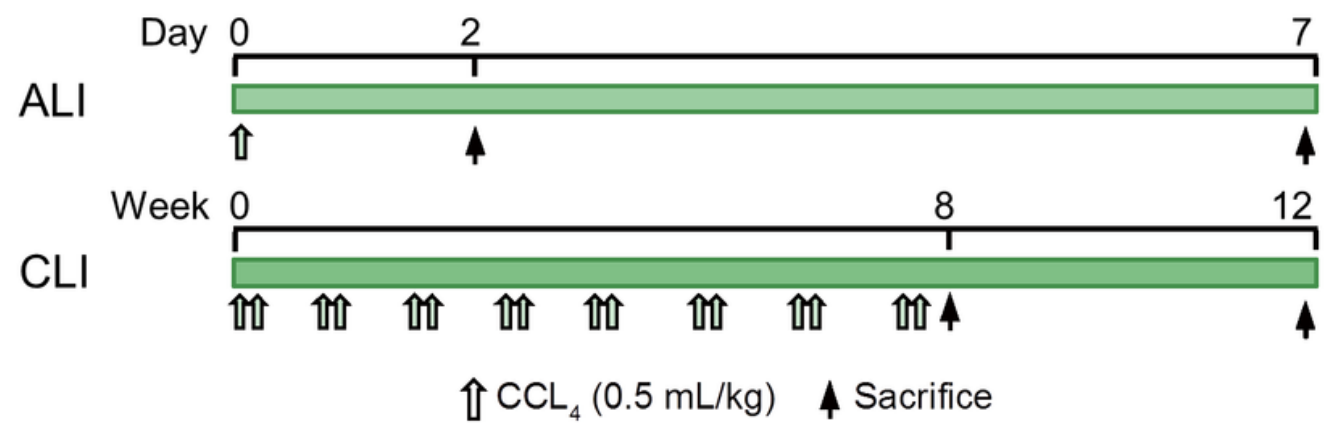

B
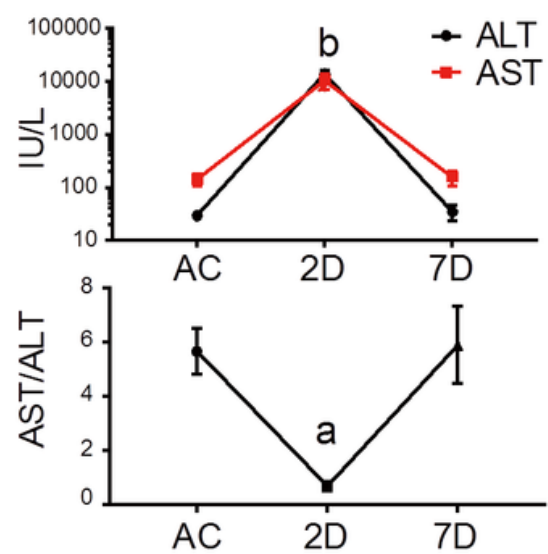

$\mathrm{D}$
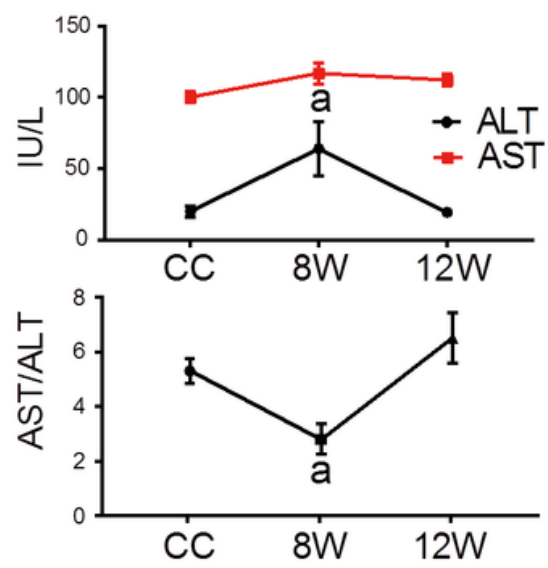

C
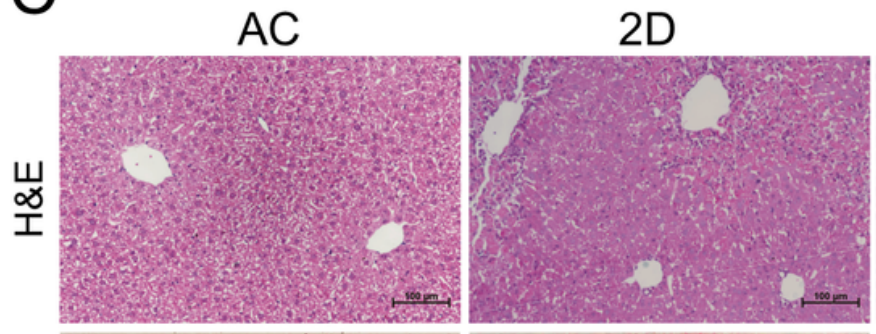

7D
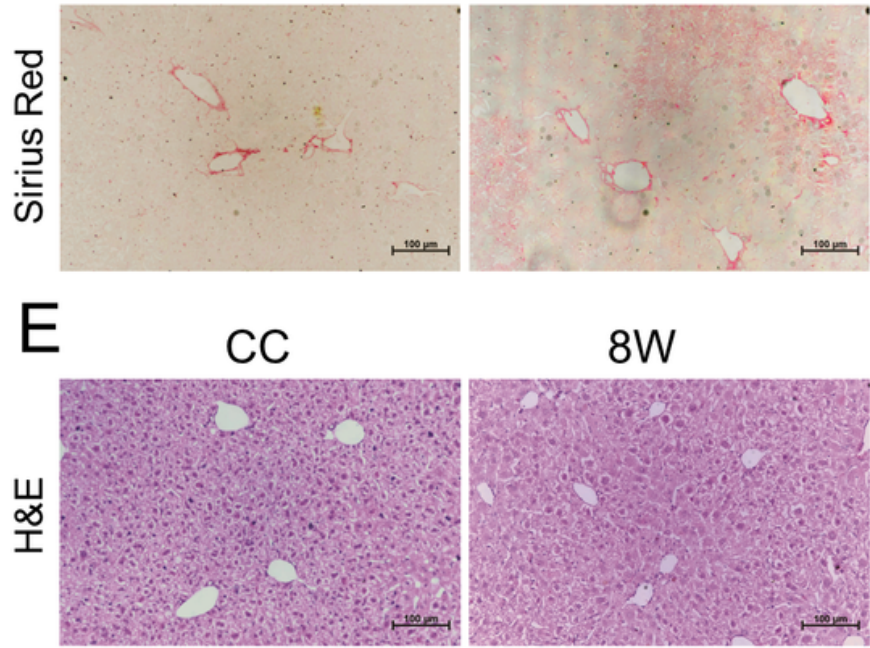

$8 \mathrm{~W}$

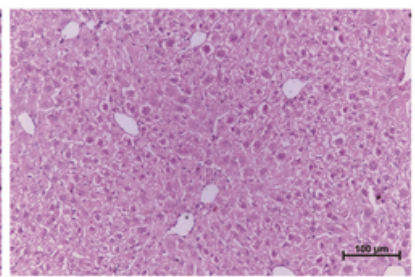

$12 \mathrm{~W}$
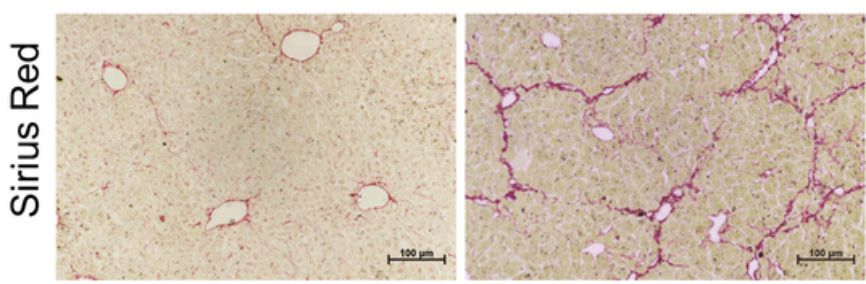
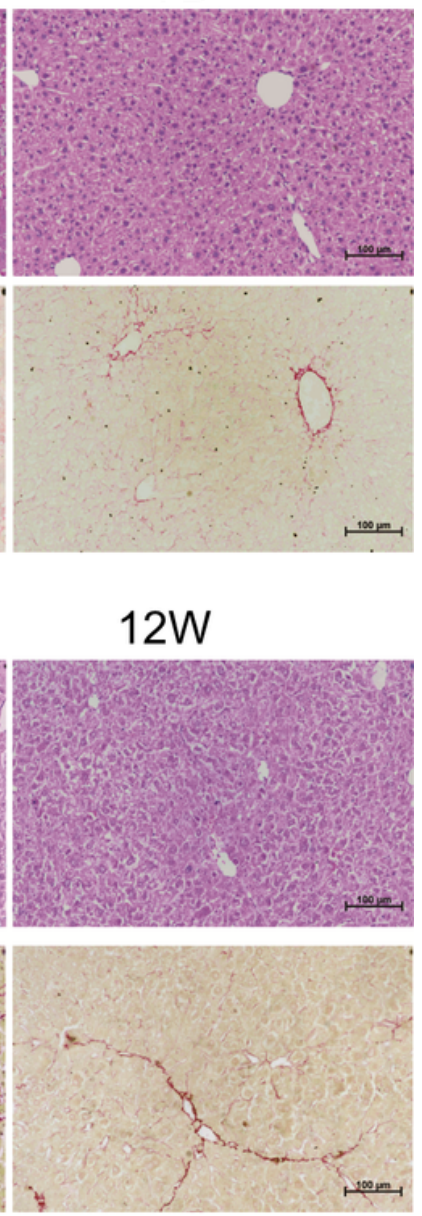

\section{Figure 1}

Establishment and validation of CCL4-induced acute liver injury (ALI) and chronic liver injury (CLI) in mice. A: Workflow for the establishment of the ALI and CLI mouse models; B: Serum ALT and AST levels in ALI mice. Compared with the AC group, a $\mathrm{P}<0.05$, b $\mathrm{P}<0.01$; C: H\&E and Sirius Red staining of liver sections from AC, 2D, and 7D mice; D: Serum ALT and AST levels in CLI mice. Compared with the CC group, a $P<0.05$; E: H\&E and Sirius Red staining of liver sections from $C C, 8 W$, and $12 \mathrm{~W}$ mice. Scale bar $=$ 100 um. D: day; W: week; AC: ALI control; CC: CLI control. 
A
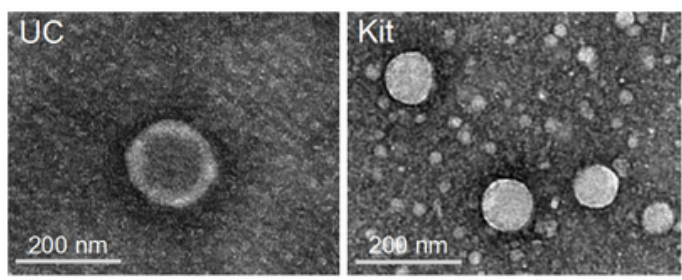

B

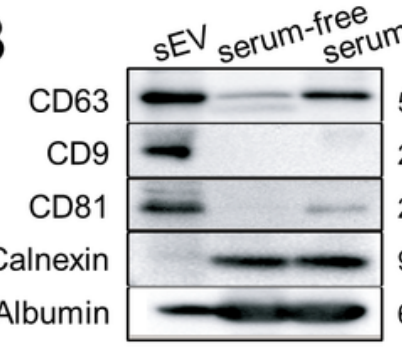

$53 \mathrm{kDa}$

$28 \mathrm{kDa}$

$26 \mathrm{kDa}$

$90 \mathrm{kDa}$

$66 \mathrm{kDa}$
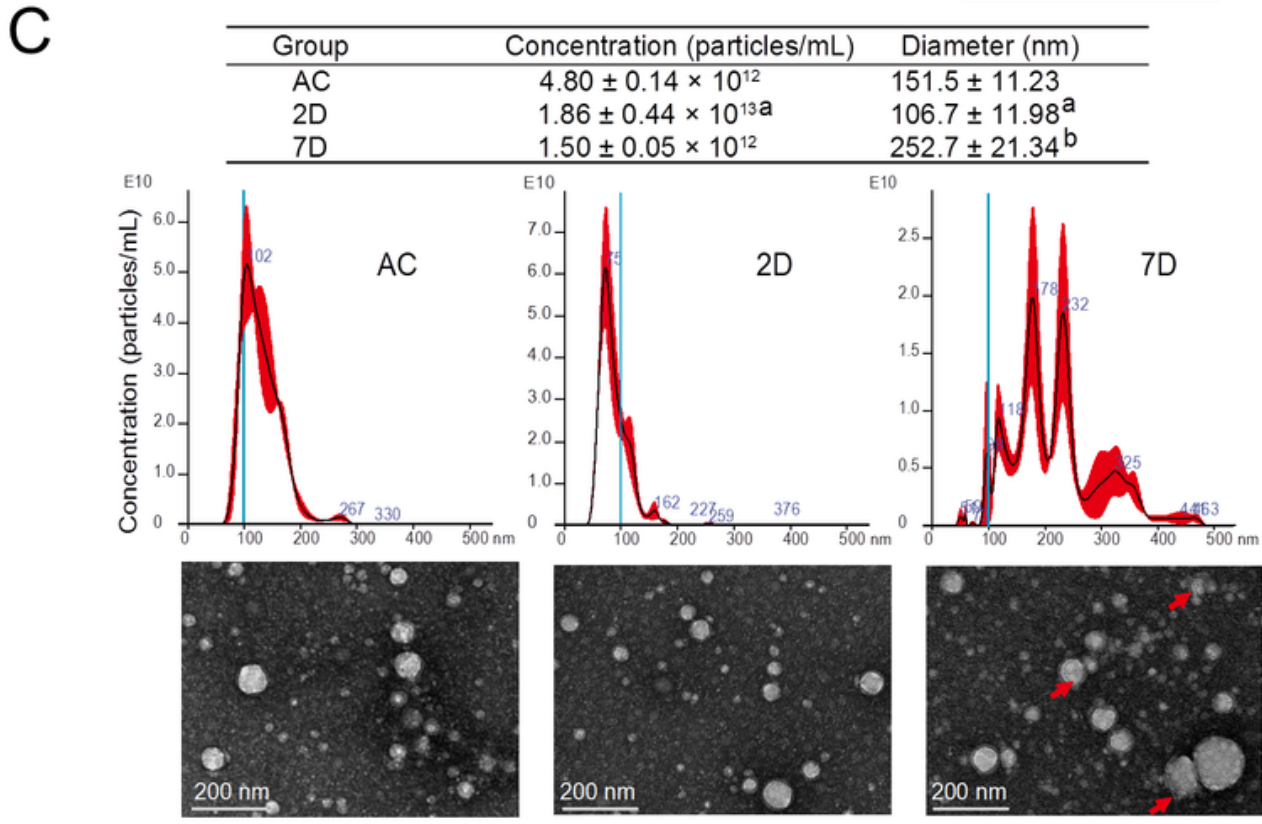

$\mathrm{D}$

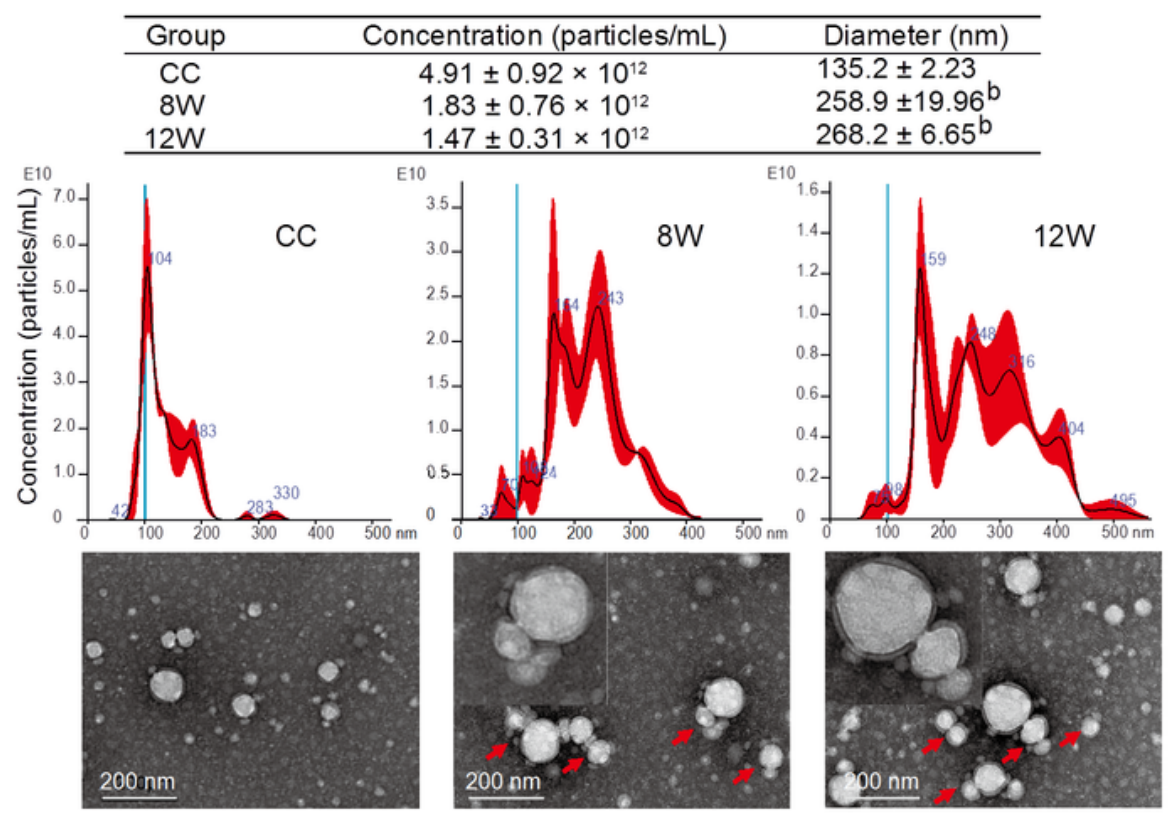

\section{Figure 2}

Characterization of isolated mouse serum small extracellular vesicles (sEVs). A: TEM images of the particles isolated using ultracentrifugation and an ExoQuick precipitation kit; B: Representative Western blotting bands for CD63, CD81, CD9, calnexin, and albumin; C: NTA plots for the size distribution and concentration of the isolated particles in each group from ALI mice and the corresponding TEM images. Compared with the AC group, a P $<0.05$, b P $<0.01$; D: NTA plots for the size distribution and 
concentration of the isolated particles in each group from CLI mice and the corresponding TEM images. Compared with the $\mathrm{CC}$ group, $\mathrm{b} \mathrm{P}<0.01$. The red arrow indicates aggregated particles; representative particles were amplified and are exemplified in the top left corner. Scale bar $=200 \mathrm{~nm}$. UC: ultracentrifuge; TEM: transmission electron microscopy; D: day; W: week; AC: ALI control; CC: CLI control.
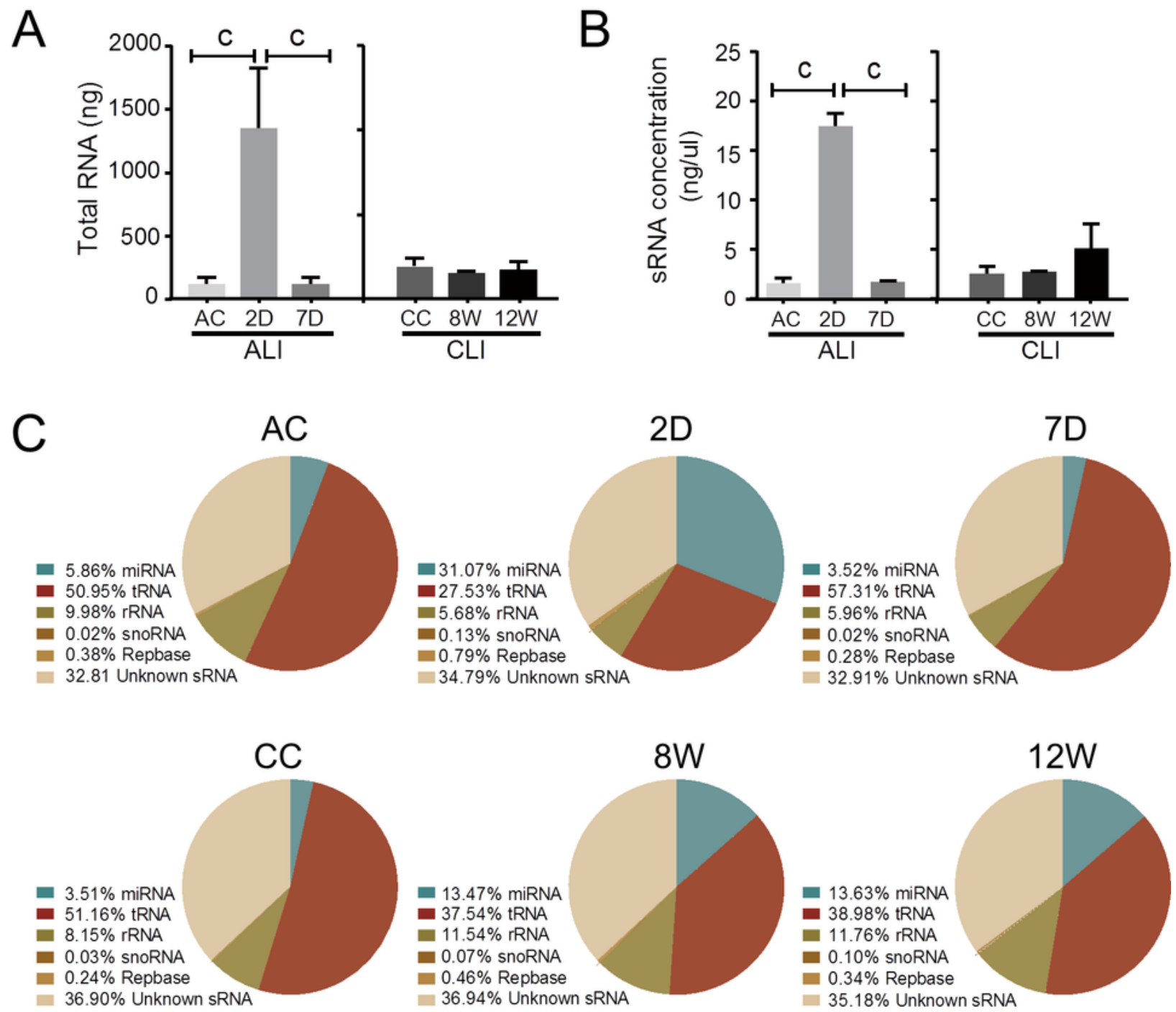

D

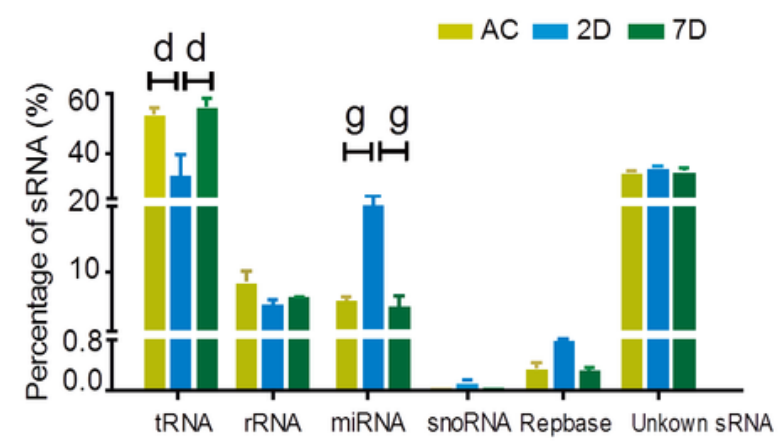

CLI

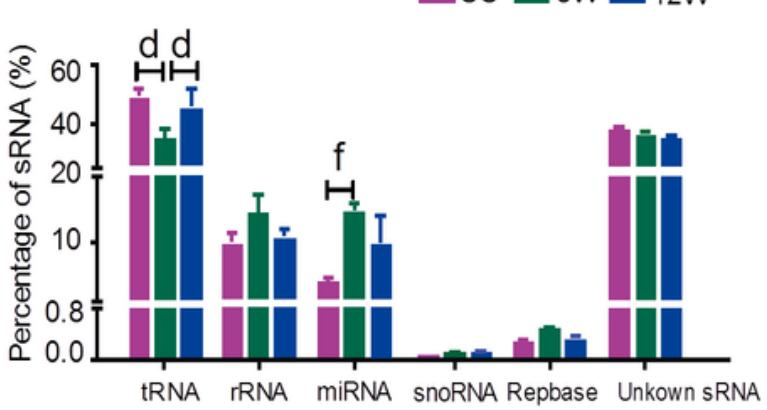

Figure 3 
Annotations of sRNA in serum sEVs from ALI and CLI mice. A: Total RNA in serum sEVs compared with the $2 \mathrm{D}$ group, $\mathrm{C} P<0.001$; $\mathrm{B}$ : sRNA concentrations in serum sEVs compared with the $2 \mathrm{D}$ group, $\mathrm{C}<<$ 0.001; C: Representative pie charts depicting the annotated sRNA species and their proportions in the different groups; D: Statistical analysis of sRNA species in sEVs. Compared with the 2D group, $\mathrm{d} P<$ 0.0001; compared with the 8W group, $\mathrm{f} P<0.01, \mathrm{~g} P<0.0001$. D: day; W: week; AC: ALI control; CC: CLI control.
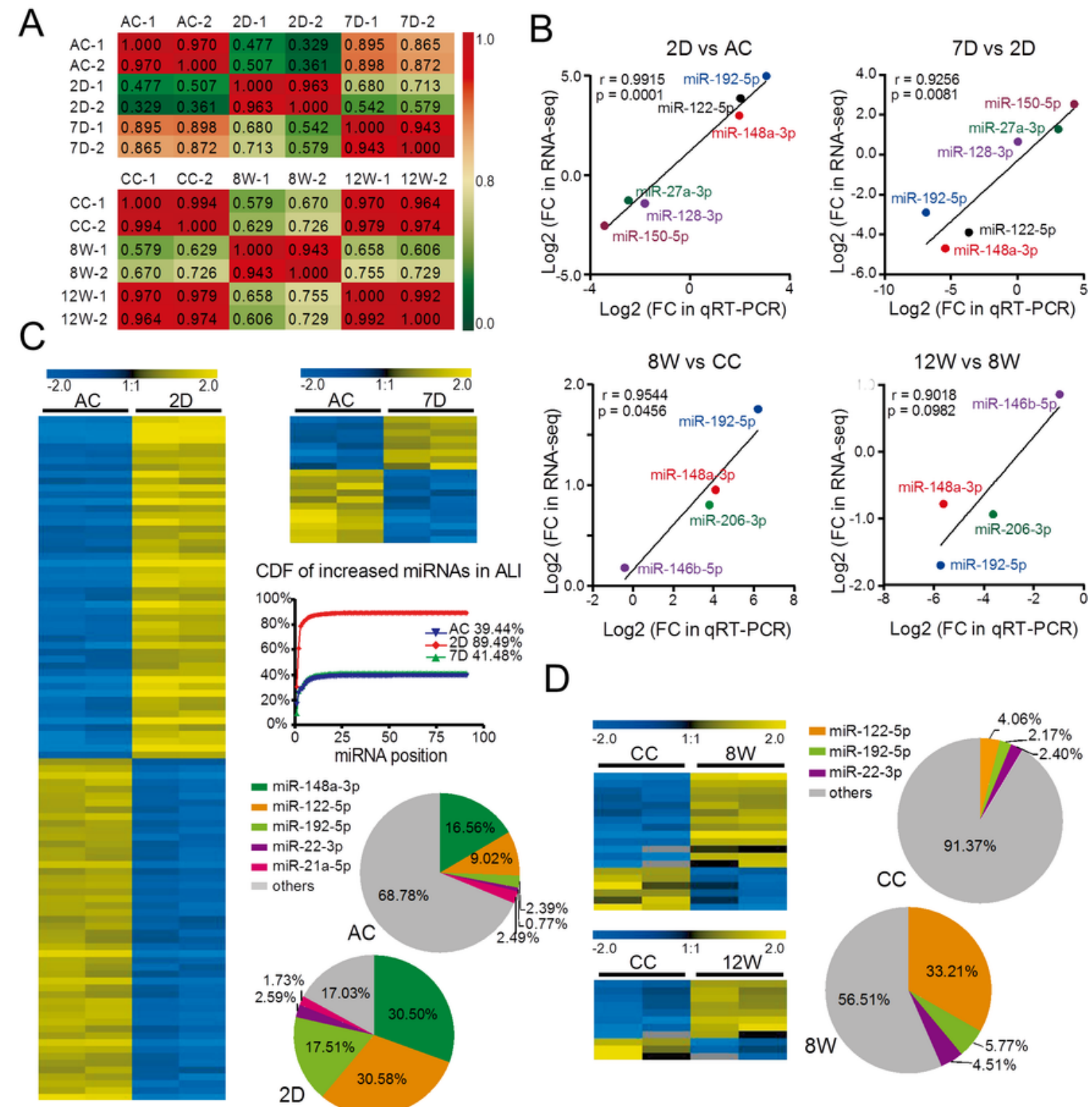

D
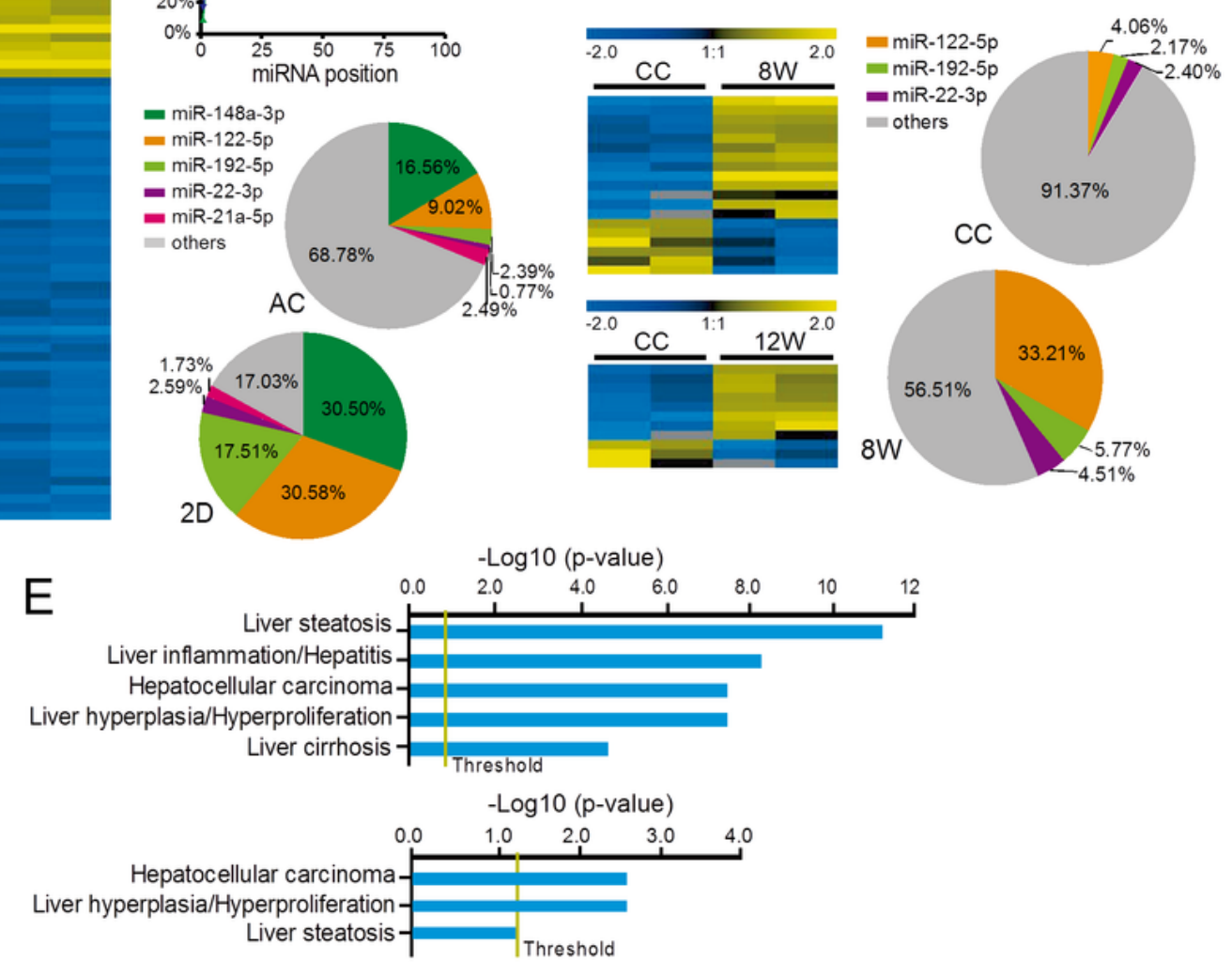
Differentially expressed miRNAs in serum sEVs from ALI and CLI mice and their biological significance. A: Pearson correlation coefficients between samples from ALI and CLI. The correlation coefficient values are labeled in the heat map; red or green represents high or low correlation, respectively; B: Validation of RNAseq data by qRT-PCR for miRNAs from ALI and CLI; C: Heatmap for the differentially expressed miRNAs in the $A L I$ injury stage (2D vs. AC) and recovery stage (7D vs. AC), a cumulative distribution frequency (CDF) plot for the increased miRNAs in ALI, and pie charts illustrating the proportions of the top five upregulated miRNAs in the AC and 2D groups; D: Heatmap for the differentially expressed miRNAs in the CLI injury stage ( $8 \mathrm{~W}$ vs. $C C$ ) and recovery stage ( $12 \mathrm{~W}$ vs. $C C)$. The pie charts illustrate the proportions of the top three upregulated miRNAs in the $\mathrm{CC}$ and $8 \mathrm{~W}$ groups; $\mathrm{E}$ : The top hepatotoxicity processes related to the differentially expressed serum sEV miRNAs in ALI (top) and the differentially expressed serum sEV miRNAs in CLI (bottom) cataloged by IPA-Tox function analysis. D: day; W: week; AC: ALI control; CC: CLI control.

$A$

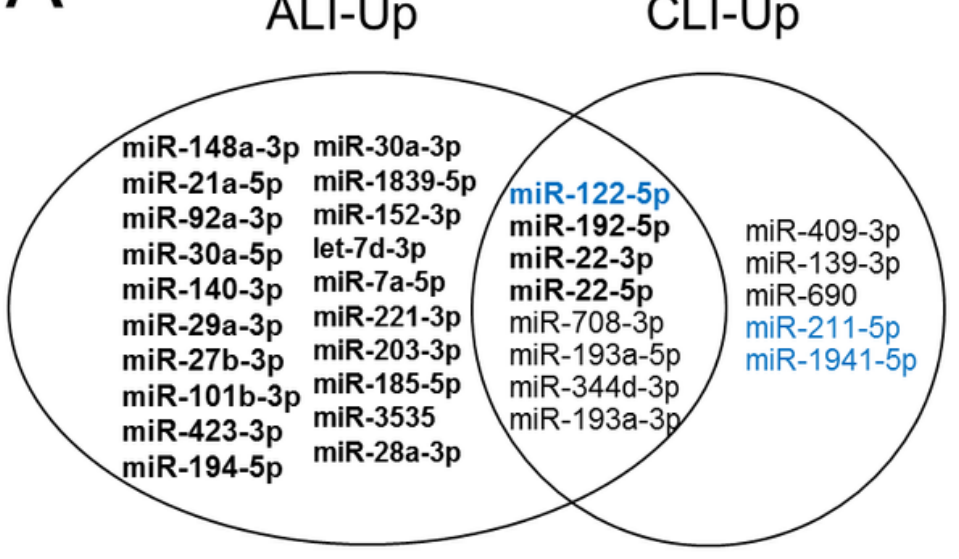

ALI-Down CLI-Down

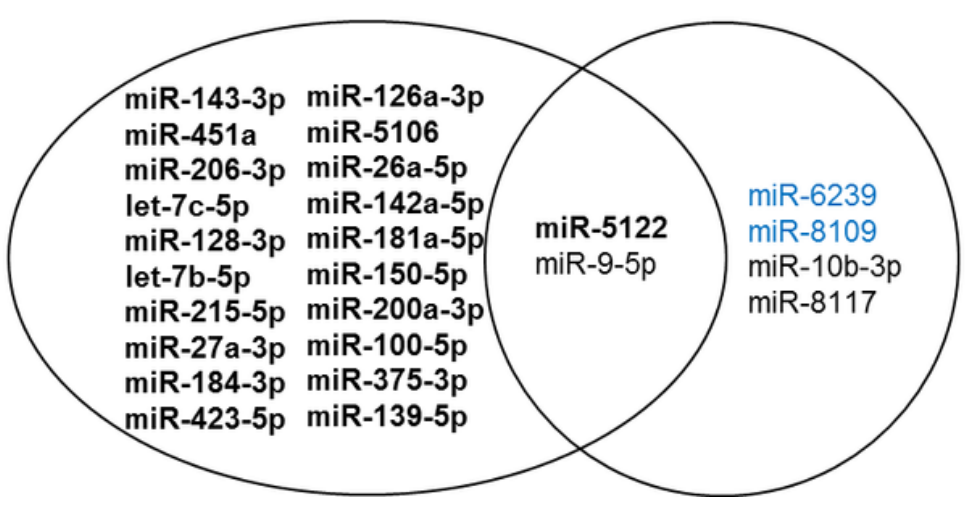

B

ALI-R-Up CLI-R-Up

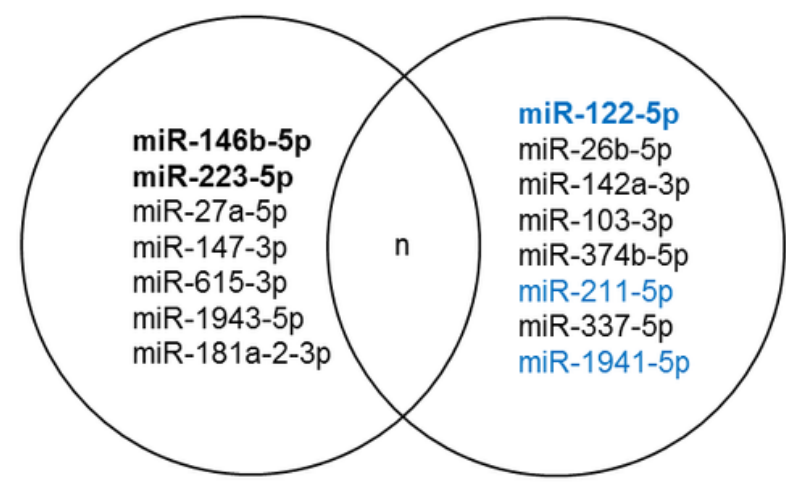

ALI-R-Down CLI-R-Down

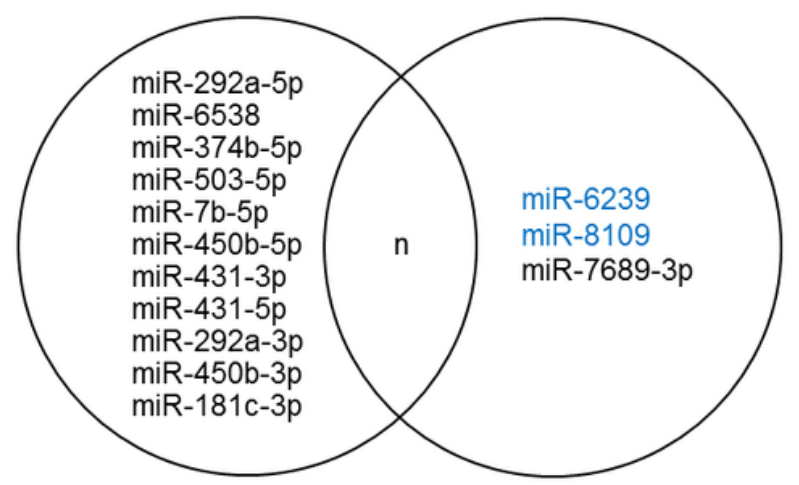

Figure 5

Common and specific miRNAs for acute and chronic liver injury and recovery in mouse models. A: Common and specific miRNAs for acute and chronic liver injury; B: Common and specific miRNAs for the recovery stages of acute and chronic liver injury. ALI: acute liver injury; CLI: chronic liver injury; ALI-R: acute liver injury recovery, CLI-R: chronic liver injury recovery; bold font: TPM Mean > 1000; blue font: overlapping miRNAs in injury and recovery; n: not applicable. 


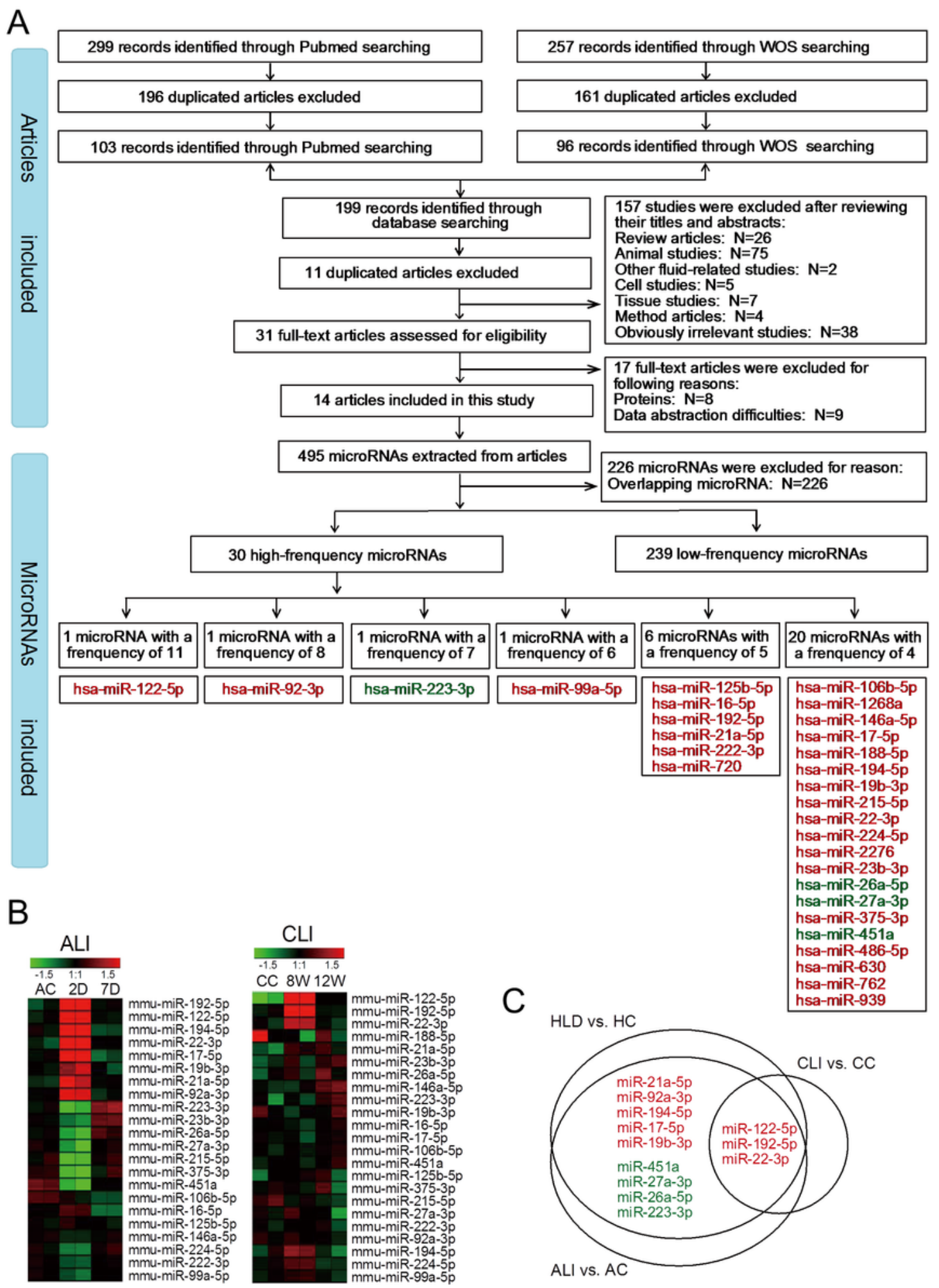

Figure 6

Differentially expressed circulating miRNAs in human liver disease (HLD) and common miRNAs in mice. A: Flow chart of study selection and miRNA screening; B: Heatmap for the expression of high-frequency HLD-related circulating miRNAs in ALI and CLI serum sEVs; C: Common miRNAs in the serum sEVs from mice with ALI and CLI and in the circulation in the context of HLDs. Red font: upregulated miRNAs; green font: downregulated miRNAs; HC: healthy control; AC: ALI control; CC: CLI control. 
A
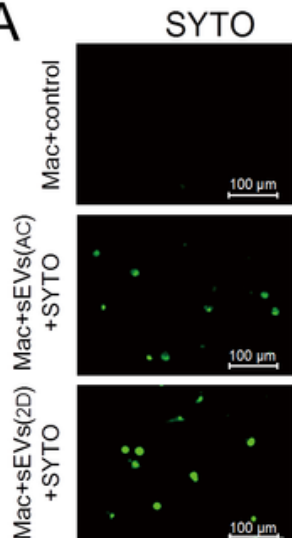

$100^{\circ} \mathrm{mm}$
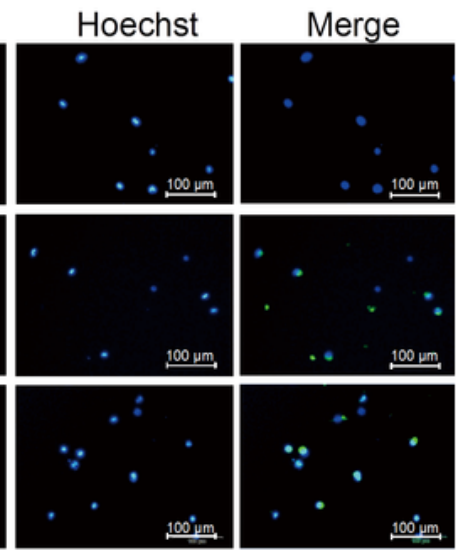

C
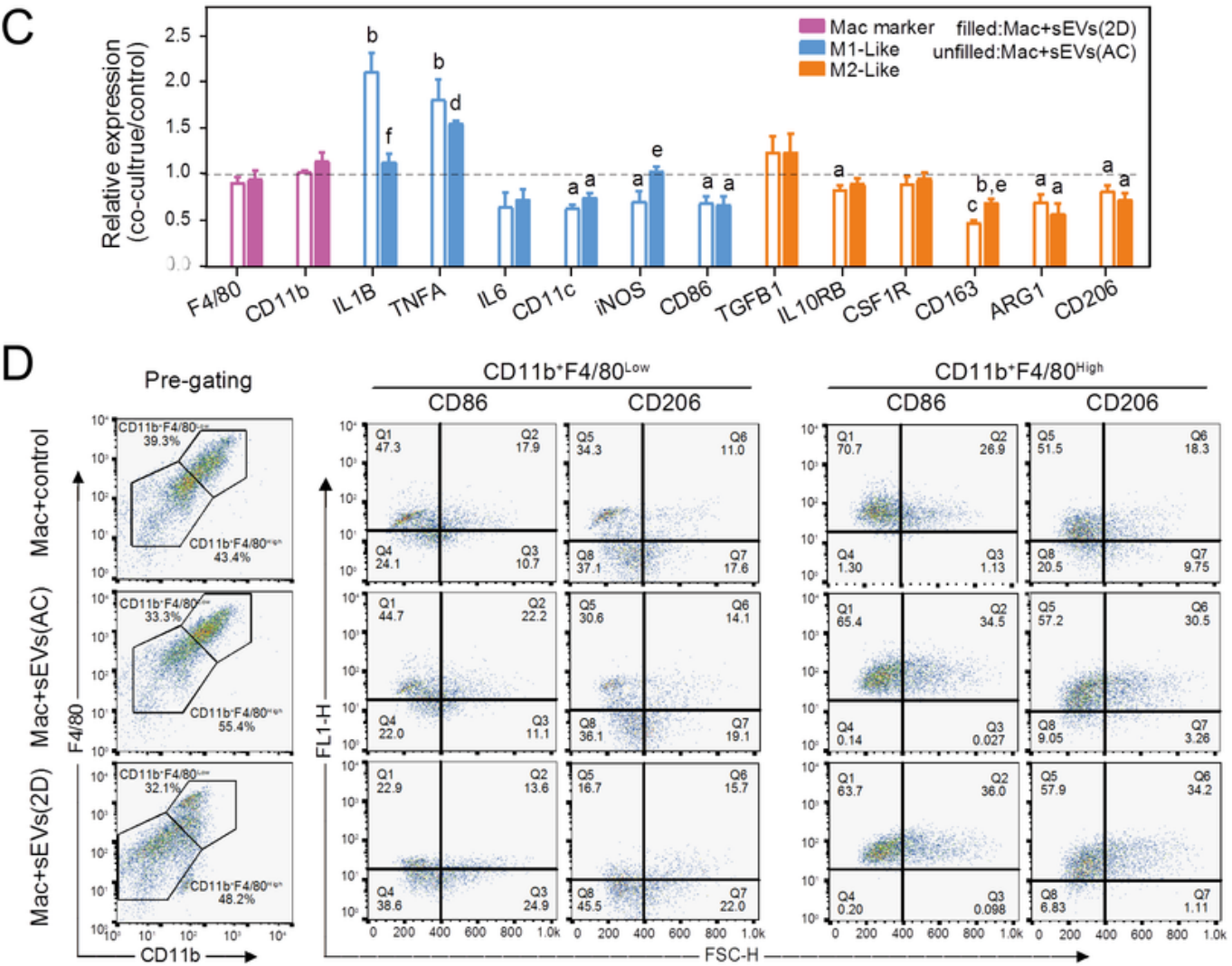

E

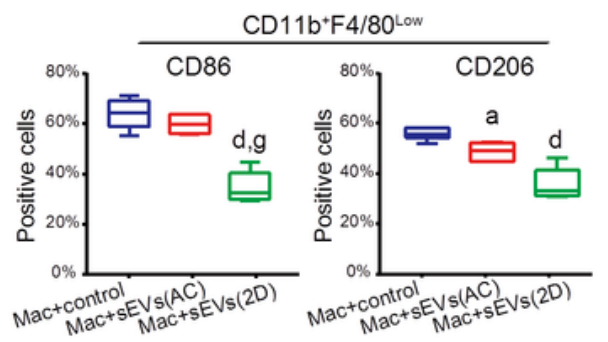

$\mathrm{F}$

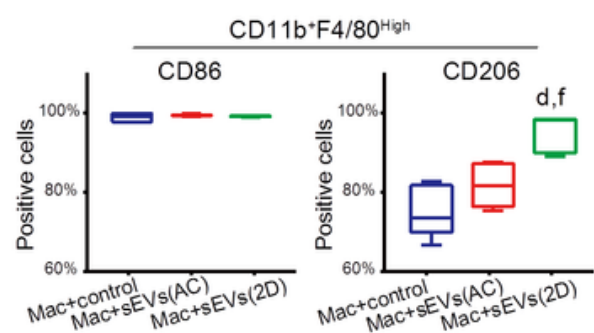

\section{Figure 7}

Uptake of serum sEVs by hepatic macrophages and subsequent reprogramming. A: Uptake of SYTOlabeled serum sEVs from normal (AC) or ALI (2D) mice by primary hepatic macrophages; B: Hepatic macrophages were incubated with AC or $2 \mathrm{D}$ serum sEVs for $24 \mathrm{~h}$. The number of attached cells per $200 \times$ field is shown; C: Expression of M1- and M2-like cell surface markers and cytokines in hepatic macrophages incubated with mice serum sEVs. The unfilled column represents macrophages incubated 
with AC sEVs, and the filled column represents macrophages incubated with 2D sEVs. Compared with the untreated control group, a $\mathrm{P}<0.05$, b $\mathrm{P}<0.01, \mathrm{c} p<0.001$, d $\mathrm{P}<0.0001$; compared with the AC sEV treatment group, e $P<0.05, \mathrm{f} P<0.01 ; \mathrm{D}$ : Macrophages were defined as $C D 11 \mathrm{~b}+\mathrm{F} 4 / 80 \mathrm{Low}$ and $\mathrm{CD} 11 \mathrm{~b}+\mathrm{F} 4 / 80 \mathrm{High}$ subgroups. The representative images show the percentage of CD86-and CD206positive cells in each subgroup subjected to the control, AC sEV, and 2D sEV treatments; E: CD86- and CD206-positive cells in each subgroup. Compared with the control group, a $P<0.05, d P<0.0001$; compared with the AC sEV treatment group, $\mathrm{P}<0.01, \mathrm{P}<0.0001$. Mac: macrophage; scale bar $=100 \mu \mathrm{m}$. D: day; AC: ALI control.

\section{Supplementary Files}

This is a list of supplementary files associated with this preprint. Click to download.

- Supplementary.rar 